\title{
Research of Design and Simulation of Polarized Reconfigurable Antenna
}

\author{
Xubao Sun*, Yanzhuang Yin, Mingming Lv, Long Ma \\ Shandong University of Science and Technology, College of Electronic, Communication and Physics, \\ Qingdao, China.
}

Corresponding Author: Xubao Sun

Keywords: Polarized reconfigurable antenna, Axial ratio, HFSS

\begin{abstract}
Reconfigurable antenna technology is a hot topic in the field of antenna. The reconfigurable antenna can realize the functions of multiple antennas on only one antenna. In this paper, a circularly polarized reconfigurable antenna, which is one of the reconfigurable antennas, is explored. After briefly introducing the concept and characteristics of circularly polarized reconfigurable antenna, the impedance bandwidth, axial ratio and polarization direction of a circularly polarized reconfigurable antenna are analysed. Based on summarizing the research results of polarized reconfigurable antennas in recent years, the structure diagram of circularly polarized reconfigurable antennas is drawn. Subsequently, HFSS software is used to simulate the polarization reconfigurable antenna, focusing on the parameters such as standing wave, axial ratio and polarization direction. Simulation results show that the proposed method can effectively improve the antenna bandwidth without increasing the antenna volume significantly.
\end{abstract}

\section{Introduction}

With the rapid development of wireless communication systems, more and more antennas are installed in limited space. It is a great challenge to reduce the cost of wireless communication systems, to realize the miniaturization of wireless communication systems and to reduce the electromagnetic compatibility between wireless communication systems. With the increasing interference between systems, the requirements of modern communication systems are becoming more and more difficult to meet, and reconfigurable antennas emerge as the times require. Reconfigurable antennas can achieve the performance of multiple antennas using only one antenna. It has a certain inhibitory effect on the increasing interference between antennas caused by antennas. Polarization reconfigurable antenna is the key point in the research of reconfigurable antenna. It can improve space freedom and gain more gain in limited space, which can greatly improve the transmission rate and system capacity of wireless communication systems. In modern wireless communication systems, the polarization reconfiguration can also effectively reduce the multipath fading. In the mobile communication system, the timely adjustment of antenna polarization according to the characteristics of different channels will help to select the best received signal in real time because of the change of the wireless channel with time.

Each of the parameters required by the antenna, such as the working frequency, the direction, the impedance bandwidth and the polarization mode, is determined by the distribution of the radiator or the current on the enclosed surface. The realization of the reconfigurable antenna also changes the current distribution of the missile body by means of electricity and machinery to change the working characteristics of the antenna. Reconfigurable antennas appear in the case of how to use one antenna to realize the functions of multiple antennas. The diversity of antenna can overcome the signal fading caused by multipath effect, improve the communication quality and improve the channel capacity. Polarization diversity is one of the antenna diversity techniques. Aiming at the multipath propagation effect of the signal in the wireless transmission process, two polarized orthogonal antennas are used to receive the orthogonal polarization components to obtain diversity gain. At present, the development of reconfigurable antennas mainly depends on the technology of microelectromechanical systems. The different reconfigurable characteristics of the antenna are 
realized by loading the switches, switches or diodes in the proper position of the antenna aperture by controlling the different working states of these switches. The main purpose of reconfigurable antennas is to change some characteristic parameters of antennas to meet the needs of practical applications.

The realization process of the reconfigurable antenna is to reconfigure the current or radiation field of an antenna, redistribute the performance, and then change the function of the antenna. According to the performance classification obtained in the antenna design, the reconfigurable antenna can obtain four reconfigurable properties, namely, frequency reconfiguration, polarization reconfiguration, direction reconfiguration, and the mixed reconfiguration of the three. The different reconfigurable performance is applied to different situations according to the needs, when so many performance reconfigurable choices are faced. The designers who need reconfigurable antennas must decide on the reconfigurable performance of the antenna and the reconfigurable method used at the very beginning. As for reconfigurable methods, antenna designers can use all kinds of devices and techniques that can satisfy both the conditions and the performance of the antennas at the same time.

\section{Theory of Circularly Polarized Reconfigurable Antenna}

\subsection{Concept}

With the rapid development of modern radar and communication systems, all kinds of military and civilian electronic devices, represented by modern wireless communication and satellite communication, are developing in the direction of miniaturization, broadband, high efficiency, large capacity, easy installation and multifunction. Reconfigurable antenna technology should be carried out. The technology uses PIN or MEMS to change its physical structure or size dynamically, so that it has multiple antenna functions. At the same time, because of the particularity of circularly polarized and the superiority of microstrip antenna, the demand of small circular polarized microstrip patch antenna is increasing. In space applications, the system capacity can be increased by transmitting, receiving and multiplexing in the same spectrum range. Although most WLAN systems require linear polarization, eventually the use of circularly polarized will become the advantage of mobile systems. Microstrip patch antenna is a resonant microstrip patch antenna, which is the most common form of microstrip antenna. The metal patches of this antenna usually use shape - shaped graphics, such as round, rectangular, circular and circular slices, most of which are fed on the coaxial line or fed by microstrip lines, which simplify the analysis of the antenna and expect its performance. The biggest feature of this antenna is its high efficiency, the substrate thickness is much smaller than the wavelength, and it is easy to be miniaturized, but the impedance band is narrow. Although the antenna circularly polarized structure is simple and bandwidth is very large to reconfiguration, two coaxial endpoints are needed, and it is easy to cause mutual coupling and severe cross polarization. Through the loading of parasitic units, the current path is extended, to achieve multi frequency phenomenon and have good antenna characteristics.

\subsection{Features}

Linearly polarized antennas are widely used in traditional antennas because of their low cost, simple processing and flexible design. However, during the transmission of space wireless channels, the interference of some buildings or trees will inevitably produce diffraction, reflection and scattering, and polarization mismatch will inevitably exist if the linear polarization antenna is received. The results show that the power difference between the orthogonal polarization components is high and the main reason for this result is the influence of the obstacles in the transmission process. There is a serious loss of horizontal polarized waves at the receiving end of the building with rich zones and lack of buildings in empty mines. Even if the antenna placed at 45 degrees can reduce the receiving power difference when receiving, it is obtained at the cost of increasing correlation. If the circularly polarized antenna is used to receive, the relative balance of the receiving power can be obtained, and the correlation is low. The circular polarized antenna has 
the rotational orthogonality, that is, the antenna is a right-handed circularly polarized wave, and it only receives the right-handed circularly polarized wave, and does not receive the left-handed circularly polarized wave, and vice versa. The circularly polarized antenna can receive any linearly polarized wave, and its transmitted wave can also be received by any linearly polarized antenna. This is because the circularly polarized waves can be decomposed into two equal amplitude polarized waves in both time and space. Therefore, it is essential to generate two linearly polarized electric field components with orthogonal space and equal amplitude and phase difference. The circularly polarized wave belongs to the instantaneous rotation field of equal amplitude. That is, the track of the instantaneous electric field vector is a circle. If the instantaneous electric field vector rotates in the direction of the left-hand helix from the propagation direction, it is called the left circularly polarized wave. If the right-hand spiral is rotated, it is called the right-hand circular polarized wave. Arbitrary polarized waves can be regarded as the synthesis of circularly polarized waves. As a special case, two circular polarized waves with opposite rotation and equal amplitude can synthesize a linearly polarized wave. Because of this, a circular polarized antenna can receive arbitrary polarizing waves, and its radiation can be received by other arbitrarily polarized antennas. It is precisely because of this reason that circularly polarized is often used in the applications of electronic reconnaissance and interference.

\subsection{Parameters}

Impedance bandwidth. It reflects the impedance matching of antennas, generally referring to the difference between the two frequencies when the antenna performance drops to intolerable. In practical applications, when the bandwidth of the return loss $S<-10 \mathrm{~dB}$, it is usually the impedance bandwidth.

Axial ratio. The axial ratio is an important parameter to measure whether the antenna is circularly polarized. The ratio of the long axis to the short axis defined as the polarization ellipse:

$\mathrm{AR}(\mathrm{dB})=20 \lg \left(\frac{A}{B}\right)$. The basic electrical parameters of a circularly polarized antenna generally refer to the axial ratio of its maximum gain direction. For pure circular polarized waves, we have AR $=1$. In the antenna design, the bandwidth that satisfies $A R \leq 3 \mathrm{~dB}$ is defined as the circularly polarized of the antenna.

Polarization direction. The antenna polarization direction is a parameter that describes the direction of the electromagnetic wave vector space of the antenna radiation. According to the Maxwell equation, the electric field has a certain relationship with the magnetic field, and the direction of the space of the electric field vector in the maximum direction of the antenna is generally used as the polarization direction of the antenna. The antenna can be divided into linear polarization antenna, circular polarized antenna and elliptical polarized antenna.

\section{Design of Polarized Reconfigurable Antenna}

\subsection{Design principles}

Any circular polarization wave can be decomposed into two equal amplitude linear polarization waves in space and time. Thus, the basic principle of realizing circular polarization is obtained: two space orthogonal polarized electric field components of equal amplitude and phase difference are produced. Although the form of circular polarized antenna is different, the mechanism is not separated from its origin, and the microstrip antenna is also a circular slot structure microstrip antenna which etched a circular slot on the ground floor of the microstrip antenna and used microstrip line to feed the antenna with non-contact coupling. This antenna structure has a wider bandwidth than ordinary microstrip antennas, usually more than 5\%. By adding some structure above the gap of the ring, it is possible to further expand the bandwidth. Therefore, the antenna with this structure has good application value. Some of the Polarization Reconfigurable schemes mentioned above are based on the ring gap structure. When working in this state, the antenna gains the maximum in the direction perpendicular to the plane of the ring gap, that is, the antenna main lobe points to this direction. The annular slot antenna works in the mode. In this mode, the antenna 
has a zero limit in the direction perpendicular to the plane of the ring joint. The maximum radiation direction is the end - shooting direction, and the gain perpendicular to the plane in the ring seam is zero. The antenna can also work in a higher order mode. However, with the increase of the mode, the surface and high mode excitation of the antenna will seriously deteriorate the axial ratio of the antenna. If a good axis ratio is obtained, the antenna should work in the lower order mode as much as possible. The higher order mode of girth antenna is not without merit. The more obvious advantage is that the antenna size can be reduced, and the antenna will be miniaturized. Considering that my design needs a good axial ratio bandwidth, these factors are integrated to determine the antenna working in the base mode state, and some other methods are used to minimize the size of the antenna.

\subsection{Design process}

The circular arc parasitic unit is loaded on the circular radiant plate. The loading position of the parasitic unit is determined by the current characteristic of the base mode TM11 of the circular patch microstrip antenna. To realize the orthogonal mode with the phase difference of 90 degrees and the same amplitude, the circular polarization is realized. The switch control parasitic unit is capacitive or inductive to realize circular polarization reconfiguration. Because the parasitic unit is outside the patch, it is easy to control and the cross polarization is small. At the same time, the current path is increased, and it also plays a certain role in reducing the antenna size. At the same time, the orthogonal slot at the 45 degrees of the feed point is loaded in the patch centre, and the current on the circular patch is segmented, so the antenna miniaturization and broadband effect can be realized by increasing the dual methods of the current path and the bend current path. Moreover, because the current intensity of the centre part of the circular patch is zero, the impedance matching is easy to achieve, and it also promotes the realization of the circular polarization of the antenna. By selecting the relative dielectric constant and thickness of the substrate, the initial circular patch size with frequency needed can be obtained through the above two expressions. The radius of the patch calculated by the above relationship is larger than the actual patch radius, which is due to the reason that the surface current of the patch becomes longer on the patch, and the error can be solved. Using the method of reactance loading, the circular microstrip antenna can realize two adjacent modes of orthogonally polarized wave. According to the parameter design principle of circular patch antenna and the method of loading the circular arc parasitic unit on the patch, the size of the final microstrip antenna is as follows: the radius of the circular plate is $15 \mathrm{~mm}$ and the size of the floor is $60 \mathrm{~mm} \mathrm{x}$ $60 \mathrm{~mm}$. The width of the parasitic circular arc is $1 \mathrm{~mm}$, and the arc is 180 degrees. The planar structure of the designed microstrip antenna is shown in Figure 1.

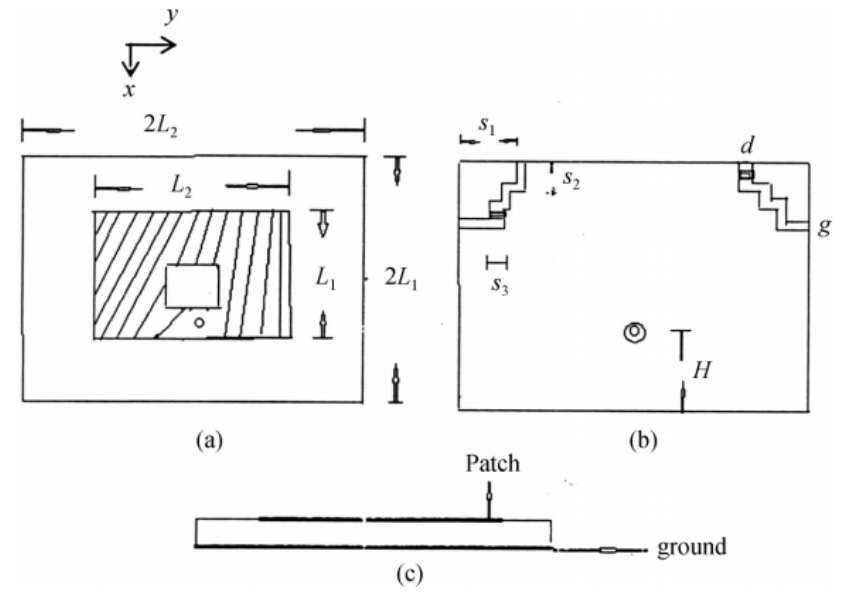

Figure 1. Structure of circularly polarized reconfigurable antenna 


\section{Simulation of Polarized Reconfigurable Antenna}

\subsection{Simulation software}

HFSS is the abbreviation of High Frequency Structure Simulator. It is the first commercial threedimensional electromagnetic simulation software in the world. It is recognized as the industry standard for the design and analysis of three-dimensional electromagnetic fields. HFSS provides a simple and intuitive user design interface, an accurate adaptive field solver, a powerful post processor with an unprecedented power analysis capability, which can calculate the parameters and full wave electromagnetic fields of a three-dimensional passive structure with arbitrary shapes. Due to the introduction of perturbation element, it is possible to design an electric field with the same amplitude and phase difference at a certain frequency. Two modes of the antenna. The radiation mechanism of antennas can be explained by cavity mode theory. When an ordinary rectangular patch antenna is fed along diagonal lines, the same frequency point will be simultaneously activated. The resonant frequency of the two modes is changed at the same time due to the influence of current interruption on the diode control branch. When the switch is switched on and the switch is disconnected, the current of the mode can be passed through the switch, the resonant frequency is reduced, and the current of the mode cannot pass through the switch, then the resonant frequency is relatively high. If the frequency of the two designs is properly designed, the sum of the modulus and the frequency at the centre frequency should be calculated.

\subsection{Simulation of stationary wave}

The two waves, which have the same amplitude and the opposite direction, form a distribution state along the transmission line. One of these waves is usually the reflection of another wave. A wave node appears at the point where the two voltages increase, forming a wave node at the point where the two voltages are subtracted. In waveforms, the position of wave nodes and wave nodes is invariable. Antenna mismatch, antenna capacitive detuning, antenna resonance, but feeding point is not suitable. On the impedance diagram, each voltage value is a circle with infinite number of points. There are many possibilities for the state of the antenna system when the number is pressed. The circular polarization centre frequency of the Reconfigurable Microstrip antenna unit is $\mathrm{f}_{0}=$ $4.7 \mathrm{GHz}$. The simulation data of the reflectivity curve of the antenna in the left and right circular polarization state are shown in the figure.

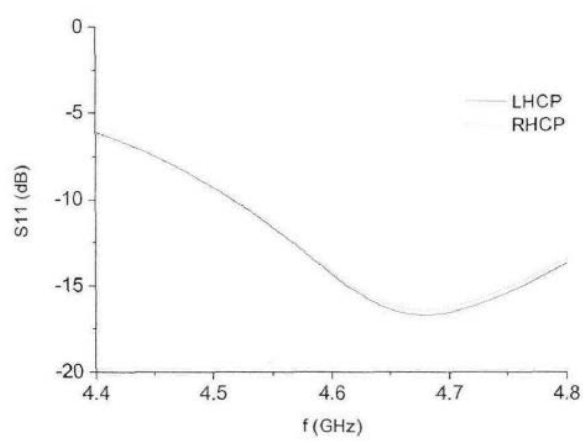

Figure 2. Coefficient curve of antenna Reflection in the left and right circular polarization state

\subsection{Simulation of axial ratio}

At the centre frequency $\mathrm{f}_{0}=4.7 \mathrm{GHz}$, the antenna axis is $\mathrm{AR}=0.8 \mathrm{Db}$. When the antenna axis ratio is less than $3 \mathrm{~dB}$, the bandwidth is about $130 \mathrm{MHz}$. The simulation data of the axis ratio curve of the antenna in the left and right circular polarization state is shown. Along the direction of propagation, the path of the end of the field vector of the electromagnetic wave synthesis is a circle. If the equal amplitude and quadrature phase are not satisfied, it is elliptical polarization, that is, the path of the end sweep is ellipse. The ratio of the long and short axis is called the axial ratio. The axial ratio bandwidth is the working frequency band of the antenna which is lower than a certain value in the main radiation direction or a beam width. Circular polarized omnidirectional antenna 
has attracted more and more attention in modern wireless applications due to its own performance characteristics.

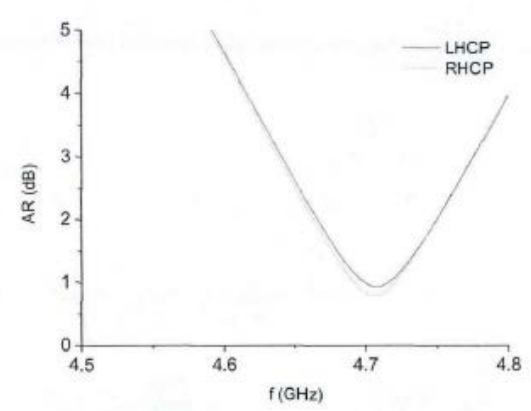

Figure 3. Axial ratio curve of polarized reconfigurable antenna

\subsection{Simulation of antenna direction}

It can be seen from the simulation curves that the corresponding characteristics of RHCP and LHCP antennas are basically the same, which provides a possible condition for the design of polarization reconfigurable antennas. Directional reconfigurable antenna is a reconfigurable antenna that changes the antenna's direction by changing the structure of the antenna without changing its frequency and polarization mode. The traditional method of changing antenna radiation pattern is mainly using phased array antenna technology, but the feeding system is complex, the distance between array elements is large, and the cost of system increases with the increase of frequency, which makes the performance of the system limited. The directional reconfigurable antenna can overcome these shortcomings of traditional phased array antennas, making the antenna structure relatively simple and easier to control. This antenna means that two or more than two or more of the characteristic parameters such as frequency, direction and polarization of the antenna can be reconstructed simultaneously.
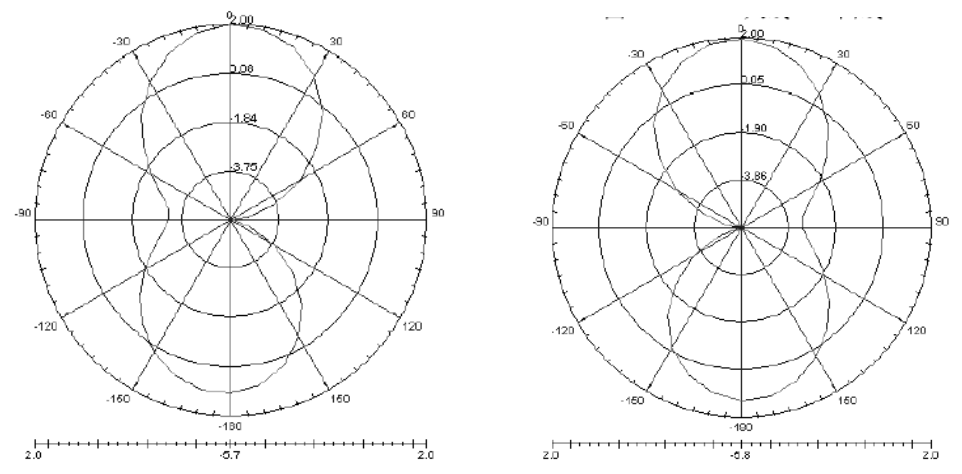

Figure 4. System modules design LHCP (left) and RHCP (right)

\section{Conclusion}

The main object of this paper is the reconfigurable antenna. Through simulation, we get some experience and conclusions as follows:

(1) The design principle and design process of circularly polarized reconfigurable antenna are analysed in this paper.

(2) This paper summarizes the notices in the design process of circularly polarized reconfigurable antennas and studies the effect of antenna structure on the performance of antennas.

(3) HFSS software is used to simulate the polarization reconfigurable antenna. The parameters such as standing wave, axial ratio and polarization direction are emphatically analysed. The results show that the antenna bandwidth can be effectively improved without increasing the antenna volume significantly. 


\section{References}

[1] Zhu H L, Cheung S W, Liu X H, et al. Design of polarization reconfigurable antenna using metasurface[J]. IEEE Trans. Antennas Propag., 2014, 62(6): 2891-2898.

[2] Lin W, Wong H. Wideband circularly polarized reconfigurable antenna[J]. IEEE Transactions on Antennas and Propagation, 2015, 63(12): 5938-5944.

[3] Ji L Y, Qin P Y, Guo Y J, et al. A wideband polarization reconfigurable antenna with partially reflective surface[J]. IEEE Trans. Antennas Propag, 2016, 64(10): 4534-4538.

[4] Wong H, Lin W, Huitema L, et al. Multi-polarization reconfigurable antenna for wireless biomedical system[J]. IEEE transactions on biomedical circuits and systems, 2017, 11(3): 652-660.

[5] Kandasamy K, Majumder B, Mukherjee J, et al. Low-RCS and polarization-reconfigurable antenna using cross-slot-based metasurface[J]. IEEE Antennas and Wireless Propagation Letters, 2015, 14: 1638-1641.

[6] Osman M N, Rahim M K A, Hamid M R, et al. Compact dual-port polarization-reconfigurable antenna with high isolations for MIMO application[J]. IEEE Antennas and Wireless Propagation Letters, 2016, 15: 456-459.

[7] Row J S, Wei Y H. Wideband Reconfigurable Crossed-Dipole Antenna With Quad-Polarization Diversity[J]. IEEE Transactions on Antennas and Propagation, 2018, 66(4): 2090-2094.

[8] Di Palma L, Clemente A, Dussopt L, et al. Circularly-polarized reconfigurable transmitarray in Ka-band with beam scanning and polarization switching capabilities [J]. IEEE Transactions on Antennas and Propagation, 2017, 65(2): 529-540.

[9] Cai Y M, Gao S, Yin Y, et al. Compact-size low-profile wideband circularly polarized omnidirectional patch antenna with reconfigurable polarizations[J]. IEEE Transactions on Antennas and Propagation, 2016, 64(5).

[10] Lin W, Wong H. Multipolarization-reconfigurable circular patch antenna with L-shaped probes[J]. IEEE Antennas and Wireless Propagation Letters, 2017, 16: 1549-1552.

[11] Li Z, Ahmed E, Eltawil A M, et al. A beam-steering reconfigurable antenna for WLAN applications[J]. IEEE Transactions on Antennas and Propagation, 2015, 63(1): 24-32.

[12] Row J S, Huang Y J. Reconfigurable Antenna with Switchable Broadside and Conical Beams and Switchable Linear Polarized Patterns[J]. IEEE Transactions on Antennas and Propagation, 2018.

[13] Wang R, Wang B Z, Gao G F, et al. Low-Profile Pattern-Reconfigurable Vertically Polarized Endfire Antenna With Magnetic-Current Radiators[J]. IEEE Antennas and Wireless Propagation Letters, 2018, 17(5): 829-832.

[14] Yang Y, Simorangkir R B V B, Zhu X, et al. A novel boresight and conical pattern reconfigurable antenna with the diversity of 360 polarization scanning[J]. IEEE Transactions on Antennas and Propagation, 2017, 65(11): 5747-5756.

[15] Nguyen-Trong N, Hall L, Fumeaux C. A frequency-and polarization-reconfigurable stubloaded microstrip patch antenna [J]. IEEE Trans. Antennas Propag. 2015, 63(11): 5235-5240.

[16] Liang B, Sanz-Izquierdo B, Parker E A, et al. A frequency and polarization reconfigurable circularly polarized antenna using active EBG structure for satellite navigation [J]. IEEE Transactions on Antennas and Propagation, 2015, 63(1): 33-40. 